\title{
Breast Cancer in Lampang, a Province in Northern Thailand: Analysis of 1993-2012 Incidence Data and Future Trends
}

\author{
Somkiat Lalitwongsa ${ }^{1}$, Donsuk Pongnikorn ${ }^{1}$, Karnchana Daoprasert ${ }^{1}$, Hutcha \\ Sriplung $^{2 *}$, Surichai Bilheem ${ }^{2}$
}

\begin{abstract}
Background: The recent epidemiologic transition in Thailand, with decreasing incidence of infectious diseases along with increasing rates of chronic conditions, including cancer, is a serious problem for the country. Breast cancer has the highest incidence rates among females throughout Thailand. Lampang is a province in the upper part of Northern Thailand. A study was needed to identify the current burden, and the future trends of breast cancer in upper Northern Thai women. Materials and Methods: Here we used cancer incidence data from the Lampang Cancer Registry to characterize and analyze the local incidence of breast cancer. Joinpoint analysis, age period cohort model and Nordpred package were used to investigate the incidences of breast cancer in the province from 1993 to 2012 and to project future trends from 2013 to 2030. Results: Age-standardized incidence rates (world) of breast cancer in the upper parts of Northern Thailand increased from 16.7 to 26.3 cases per 100,000 female population which is equivalent to an annual percentage change of $2.0-2.8 \%$, according to the method used. Linear drift effects played a role in shaping the increase of incidence. The three projection method suggested that incidence rates would continue to increase in the future with incidence for women aged 50 and above, increasing at a higher rate than for women below the age of 50 . Conclusions: The current early detection measures increase detection rates of early disease. Preparation of a budget for treatment facilities and human resources, both in surgical and medical oncology, is essential.
\end{abstract}

Keywords: Breast cancer, cancer incidence - joinpoint - age period cohort model - Nordpred - Lampang - Thailand

Asian Pac J Cancer Prev, 16 (18), 8327-8333

\section{Introduction}

Decreasing mortality rates due to infectious diseases along with increasing incidence and prevalence rates of chronic and metabolic conditions, including cancer are observed as a consequence of an epidemiologic transition in Thailand in the past few decades.

New cancer cases and deaths, in less developed countries are estimated to contribute for $57 \%$ and $65 \%$ of the world cancer burden, whilst new cancer cases are expected to rise to $63 \%$ in less developed countries (Globocan 2012 - Home, n.d.). Breast cancer poses a particular problem over the coming decades as less developed countries are increasingly adopting the characteristics of a Western lifestyle, including less children per family, use of hormonal contraceptives, and increased consumption of meat, fat and dairy products.

There is a strong association between Western lifestyle factors, such as diet and parity, and the incidences of breast cancer (Layde et al., 1989; Althuis, 2005; Cui et al., 2007; Kruk, 2007). Voluntary mammographic screening is limited to women who can financially access it, and those who have an indication to undergo screening. The method of breast self examination is affordable, and is promoted through the country-wide health care network by the Ministry of Public Health. Thus, to target resources for breast cancer prevention and control, accurate incidence predictions in the future are crucial.

Breast cancer incidence rates are increasing throughout Thailand (Sriplung et al., 2006; Khuhaprema et al., 2013). The estimated age-standardized incidence rate (ASR) of breast cancer in Thailand increased from 13.5 to 30.7 cases per a 100,000 female population in 1990 and 2008 (Vatanasapt et al., 1995; Khuhaprema et al., 2013). The regions of Thailand dramatically vary in terms of population characteristics, and risk factor exposures i.e. ethnic composition and diet, and incidence rates (Khuhaprema et al., 2012).

Bordered with Myanmar and Laos, Northern Thailand's population consists of a unique ethnic and cultural make-up. As it was ruled by Burmese Kingdom for some time in the past, Burmese culture is a part of the Northern Thai culture, including the unique dietary style. Hill tribe minorities are one of the tourism attractions of this region, however, their culture has not much influenced on Northern Thai culture.

Lampang province (Lampang Province - Wikipedia, n.d.) occupies an area of 12,534.0 sq.km (Figure 1) and the

${ }^{1}$ Lampang Cancer Hospital, Lampang, ${ }^{2}$ Epidemiology Unit, Faculty of Medicine, Prince of Songkla University, Hat Yai, Songkhla, Thailand*For correspondence: hutcha.s@psu.ac.th 


\section{Somkiat Lalitwongsa et al}

population of the 2010 census was about 0.7 million. The province is subdivided into 13 districts. The main income of the province is from tourism, the ceramic industry as well as agricultural products.

Lampang Cancer Center was established in 1994 to cover cancer control activities and for the treatment of cancer patients in the upper parts of Northern Thailand. A population-based cancer registry of Lampang province was set up by the Cancer Center soon after the start of Center to provide cancer statistics for the upper region of Northern Thailand, (Health Care Service Region 1) particularly in Lampang province, where the Cancer Center is located. It was established after the registry of Chiang Mai province run by the Faculty of Medicine, Chiang Mai University.

The goal of this study was to characterize the breast cancer incidence trends in Lampang province by the calendar year, birth-cohort and age at diagnosis and to project future female breast cancer rates in the upper parts of Northern Thailand through 2030. The investigation in this study included incidence rates of female breast cancer data from the Lampang registry from the years of 1993 to 2012.

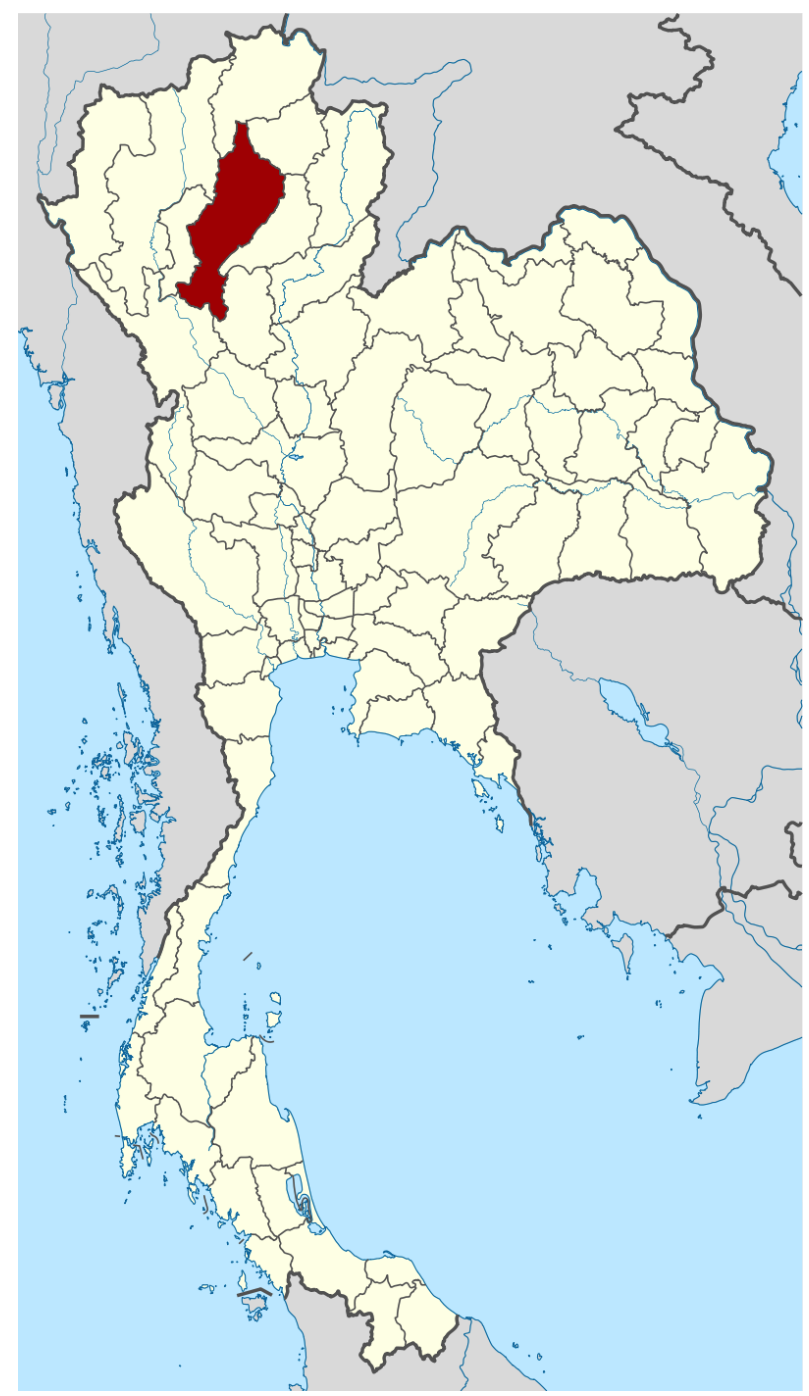

Figure 1. Map of Thailand. The province of Lampang has been highlighted

\section{Materials and Methods}

\section{Cancer registry and cancer case recruitment}

The Lampang cancer registry covers nineteen districts in the upper part of Southern Thailand. The population in Lampang province from the 2010 census was 0.74 million people of which 0.38 million were females (National Statistical Office, 2011). The registry actively ascertains cancer cases from various sources including community hospitals, private hospitals, and the Bureau of Policy and Strategies of the Ministry of Public Health.

Since the provinces of Chiang Mai and Lampang share the borders, the two cancer registries regularly exchange records of cancer patient who moves across the border for treatment in another province. In the population-based cancer registration, such the cases are registered only in the province of the first diagnosis.

The number of undetected cases is difficult to estimate due to remote villages especially in the mountainous areas with limited access to health facilities, but it is expected to be low. Female breast cancer cases were extracted from the registry database from 1993 to 2012 using ICD-10 codes C50.X. Information included age and date of diagnosis.

\section{Population denominators}

Population denominators to calculate incidence rates were estimated from the three population censuses surveyed by the National Statistical Office in 1990, 2000 and 2010 (National Statistical Office, 1992, 2011, 2002). The population denominators by both sexes for all districts were readily present in the censuses. Intercensus populations were estimated using a log-linear function between two consecutive censuses. The populations beyond 2010 were estimated, and reported by the Office of the National Economic and Social Development Board (2013).

\section{Statistical analysis}

ASRs standardized to the world population proposed by Segi, (1960), and later modified by Doll (1976), were computed for each particular year then plotted to visually illustrate the trends. Person-years used as the denominators in the computation were calculated from census data (National Statistical Office, 2011). Age-specific incidence rates were calculated for eighteen age groups ranging from 0-4 through to $80-84$, and 85 years and older and nineteen calendar periods from 1993 to 2012 (at 1-year intervals).

We evaluated trends in incidence using the JoinpointRegression Program version 4.0.4 (National Cancer Institute, 2013). Joinpoint regression identifies statistically significant trend change points (joinpoints) and the rate of change (annual percent change) in each trend segment using a Monte Carlo permutation method (Kim et al., 2000). Analyses were conducted for all females, and then for females younger than the age of 50, and females 50 years of age or older to determine the differences in incidence trends above, and below the mean age of menopause (Thomas et al., 2001; Henderson et al., 2008).

Age-period-cohort (APC) regression models were used to investigate the effects of age, the calendar year and birth-cohort on the incidence of breast cancer. Since the 
time period is only 9 years, age-specific incidence rates were calculated for 1-year rather than 5-year age groups. Although the use of 1-year intervals had the limitation of high fluctuating rates and caused wide confidence intervals, it allowed us to use the dataset within a short time period to visualize the trend, as well as being able to project the future incidence rates. We used the classical method which fits a log-linear model with a Poisson distribution to the observed data to estimate; age, period and cohort effects in a multiplicative APC model as follows:

$$
\log [R(a, p)]=f(a)+g(p)+h(c)
$$

Where the expected $\log$-incidence rates $R(a, p)$ is assumed to be equal to a linear combination of effects that adjusts for age $a$, period $p$ and birth-cohort $c$, where $c=p-a$. To address the non-identifiability problem of the APC models, two-effects models (age-period and agecohort) were first chosen, and the remaining effect (cohort or period) was then identified to the respective model's residuals using natural splines to reduce random variation (Carstensen, 2012). These are referred to as the AP-C and AC-P models. The analysis of APC models was performed using the Epi package (Carstensen et al., 2013) for $\mathrm{R}$ statistical software version 3.1.2 (R Core Team, 2014).

The Norpred package (2013) was modified for the $\mathrm{R}$ statistical software and was used to generate the projection and graph plot. Nordpred fit an APC model to the data and then calculated world-standardized incidence rates for eighteen age groups (from 0-4 to 85+, 5-year intervals) and 5-year interval periods (1993-1997, .., 2008-2012). Trends based upon all of the observed data were then extrapolated out to four 5-year periods, ending in 2030. To avoid overestimation of cases from the multiplicative model, as recommended by Olsen et al. (2008) and Mistry et al. (2011), a power of 5 function was used. The prediction model is written as:

$$
R(a, p)=\left[f(a)+D^{*} p+g(p)+h(c)\right] 5,
$$

where $R(a, p)$ is the incidence rate for age group a and period $p, f(a)$ is the age effect of age group $a, D$ is the common linear drift of period and cohort, $g(p)$ is the non-linear period effect and $h(c)$ is the nonlinear cohort effect, where $c=p-a$. We also follow the cut trend system mentioned by Mistry et al. (2011) in projection by attenuation of the linear drift by $0 D, 0.216 D, 0.483$ $D$, and $0.659 D$ respectively, for the four future 5-year projection periods.

We also applied the cut trend concept to the projection with joinpoint method with reduction of the trend in the first future 5 years by $0 \%$ and after that by geometric dampening with the factor of 0.92 (1-0.08) per year. With this method, the result was comparable to the cut trend used in Nordpred mentioned above. We used the three independent methods to project the incidence rates of breast cancer in Lampang province; joinpoint, age-periodcohort model, and Nordpred projections.

Joinpoint: Each best-fit joinpoint model was separated into its linear and residual components. The residuals described the curvature while the linear component illustrated the secular drift of the trend. APC: The linear drift $(D)$ of period and cohort parameter was identified. Nordpred: Based on the APC model, the linear drift (D) of period and cohort parameters was identified. Since we considered no abrupt change in the population such as an organized screening program in the past, we decided to use the drift of all periods in the data set, rather than the recent drift, in projecting the future trend. With the three methods, the incidence rates were extrapolated out to 2030 .

\section{Results}

A total of 2,458 female breast cancer cases were diagnosed in Lampang Province during the years of 1993 through to 2012. Of these, 1,171 occurred in females under the age of 50 and 1,287 of the cases were females aged of 50 and above. Stage distribution was shown by each age group in Figure 2. The underlying trends in stage classification, for both age groups, shows an increase in the percentage of cases for localized cancers and a decrease in metastatic cancers. A drop in unknown stage in the last 5-year period is observed.

The age group at the first peak incidence was around 45-49 in the first 5-year period, shifted to 55-59 around 2003-2007. However, it was moving back to a younger age group again in the last period (Figure 3 ). The second rise in age-specific incidence rates was observed around the age groups of 80-84 and was shifting towards younger age group around $70-74$ years.

\section{Age-period-cohort analyses and joinpoint analysis}

The AP-C and AC-P models of age-period-cohort trend analysis (Figure 4) show rough linear trends in cohort and period across the calendar years. Thus, the overall increase
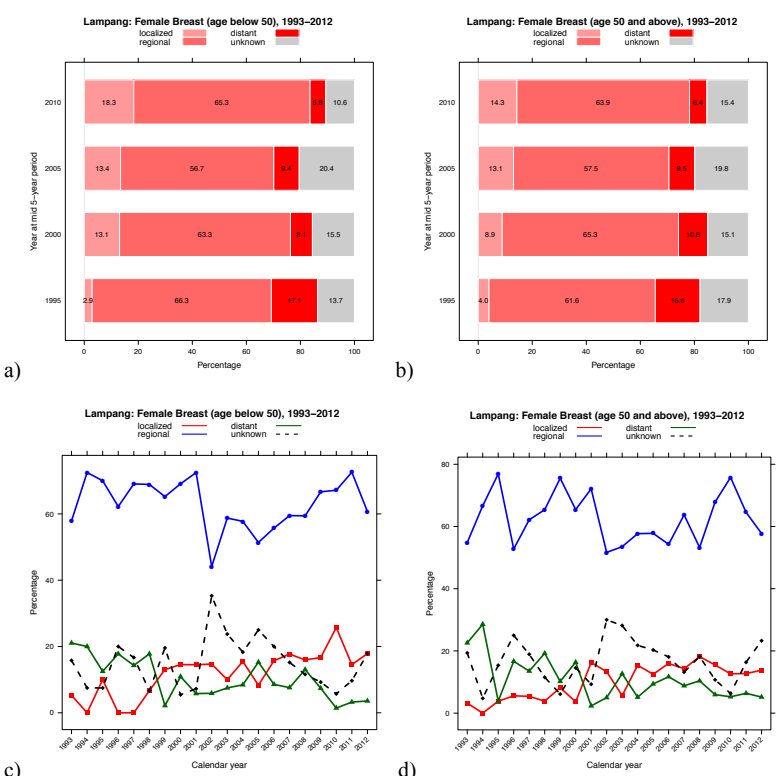

Figure 2. Stage distribution across 3-year periods for; a) women below the age of 50 and b) women at, or above the age of 50 . Percentage of stage for each year for; c) women below the age of $\mathbf{5 0}$ and d) women at or above the age of 50 


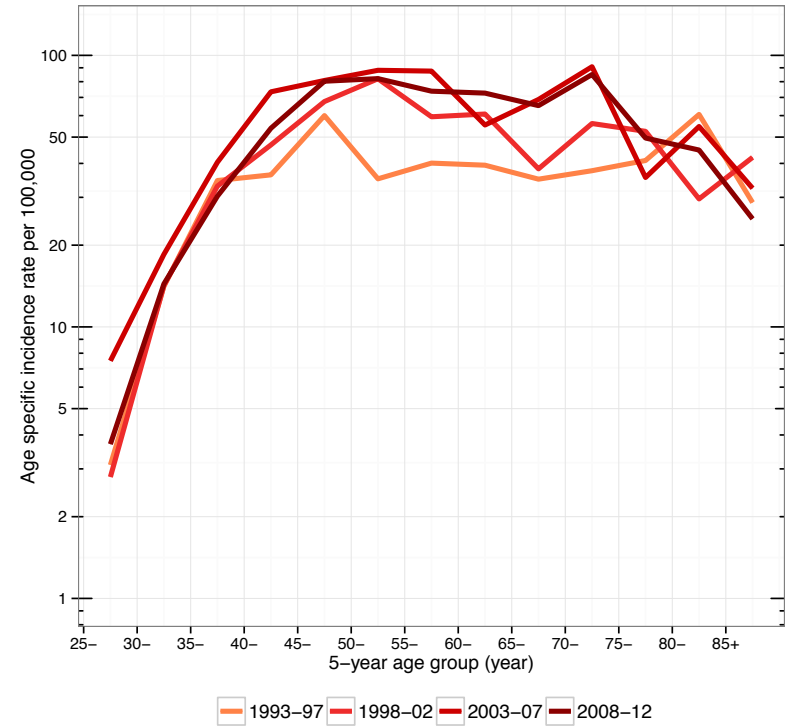

Figure 3. Age-specific incidence curves of breast cancer in Lampang in four 5-year periods from; 1993-1997, 1998-2002, 2003-2007, and 2008-2012

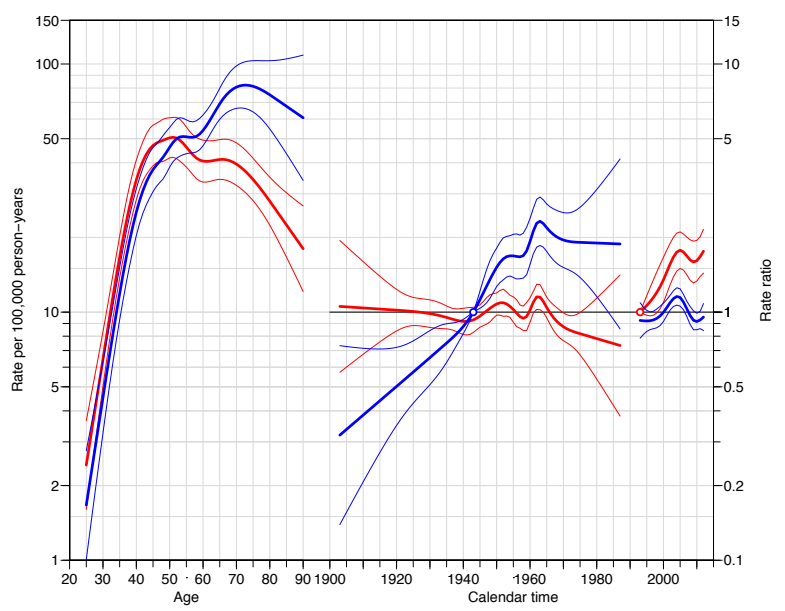

Figure 4. APC trend analysis. AC-P (blue) and AP-C (red) models for all females. Incidence rates are plotted in the log scale (left y-axis)

in incidence was explained by the age-drift model with the drift parameter (coefficient) of 0.027 and 95\%CI of $0.020-0.034$.

Breast cancer incidence rates increased from an ASR of 16.7 in 1993 to 26.3 cases per 100,000 people-years in 2012 (Figure 5). Zero join point model was the best fit for all ages and the two subgroups; women below 50 years of age, and age 50 and above. Overall, the incidence rate of female breast cancer increased at an annual percentage change (APC) of $2.7 \%$ (95\%CI: $1.6 \%, 3.9 \%)$ and $2.8 \%$ (95\% CI: $2.0 \%, 3.5 \%$ ) by joinpoint and APC models, respectively. In comparing the two models, joinpoint gave a slightly higher estimate along with a wider confidence interval than the APC models.

The projection showed that the incidence rates would be continuously increasing in the future till 2020-2025. The highest incidence might reach an ASR of 35 and 30 per 100,000 women-year by joinpoint and APC models, respectively. Similarly, both joinpoint and APC models a)

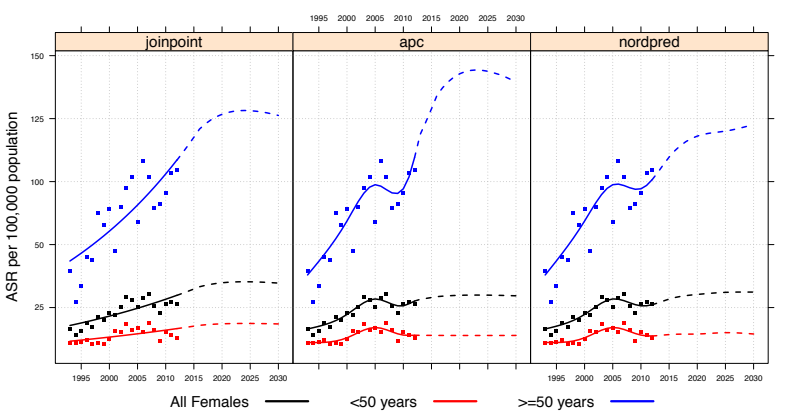

b)

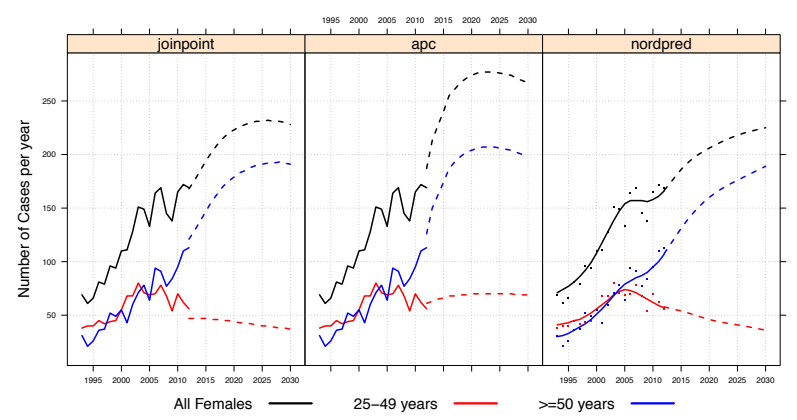

Figure 5. Trend projection of breast cancer incidence and number of cases to 2030. a) Rate projections using Joinpoint (dashed lines) and age-period-cohort (dotted lines) models both for females 50 years of age and older are much higher than those of women aged less than 50. b) Number of cases projected for all females are greater influenced by; women aged 50 years and older than those less than 50 years of age

with the cut trend method of Mistry et al. (2011), predict a plateau of ASR after 2025. Among those aged over 50, the ASR would reach 103, and 119 per 100,000 of a female population by the joinpoint and APC models, respectively.

\section{Analysis of trends by Nordpred package}

Though both Nordpred and APC methods use ageperiod-cohort model, we show the results of Nordpred separately because it bases its calculation on the 5-year not the 1-year Lexis diagram used by the APC method described above. The technique gives more stable estimates of the parameters but needs at least three 5 -year periods of observation. The annual percent change obtained by Nordpred was $2.0 \%$ (95\%CI: $1.0 \%, 4.0 \%)$. The estimate calculated from Nordpred was the lowest but the confidence interval was the widest.

\section{Number of cases}

As the proportion of elderly in Lampang also increase in the future time periods, the number of patients would reach 232, 277 and 225 cases per year by the joinpoint, APC, and Nordpred models while, the number of females over the age of 50 would reach 193, 207 and 189 (ranging from $75 \%$ to $84 \%$ of all cases) by the three methods, respectively.

\section{Discussion}

In this analysis we show that breast cancer incidence in Lampang has increased significantly since the year 
2004. This is likely due to a combination of changes in demographics, the risk profile of the population as well as, increases in surveillance and breast cancer awareness.

Projections from the joinpoint regression model to the year 2030 shows that the burden of breast cancer in the province will continue to rise in the near future, potentially reaching an ASR of 34.7, 29.7, and 31.1 cases per 100,000 person-years for all females which correspond to a total of 228,267 , and 225 cases, respectively. We prefer a continuation of the highest estimates of ASR at around 2027 for joinpoint and APC models and that of 2030 for Nordpred which were 35.2, 30.0 and 31.1, respectively. The estimated number of cases obtained from joinpoint method was not so accurate because we based the calculation on multiplying the truncated ASRs with the sum of all populations under 50 and 50 year and above accordingly. This method gave crude estimates of number of cases in the two groups, on the other hand, we could obtain the estimated number of cases by 5 -year age group from the APC and Nordpred methods. Though Nordpred method gave a little higher ASR but APC model gave much higher expected number of cases because APC model used exponential projection while we forced Nordpred to use power 5 projection method.

In summary, the ASR estimated from the three models are rather similar around 30.0 to 35.2 per 100,000 womenyears and the estimated number of cases would be between 225 to 267 cases in 2030 . Though the numbers are subject to error in estimation, we are quite sure that the estimated ASR of breast cancer in Lampang will be around a half of the incidence in western countries (Globocan 2012 Home, n.d.). Mistry et al. (2011) showed similar trends of breast cancer in the UK across age groups below 50 and 50 years and above to reach the peak around 2020 and the decline was pronounced in older age groups. So the phenomenon might not be unique to Thailand but seems to happen in Western countries as well.

The increase in ASR could be partly affected by future healthcare planning and other cancer burden control measures which, increase the early cancer detection rate by screening measures such as the use of self breast examination techniques and mammography. Increased awareness of breast cancer in the population could be playing a role and the decrease in stage of the disease would be the effect of both awareness and such screening measures. While a strong risk factor for breast cancer in a population is the number of children per family (Colditz and Rosner, 2000), it is clear that the total fertility rate (TFR) of Thai population has continuously dropped from 6.3 in 1965 to 1.6 or 1.4 in 2010 depending to the data source (Prasartkul and Vapattanawong, 2012; The World Bank, n.d.). Such the drop in TFR can mainly explain the increase in breast cancer incidence. According to the World Bank and other sources, the TFR of Thailand has been below 2 since the early 1990 and should be steady around 1.4 through 2020 and beyond, thus, this can explain the deceleration of the increase in breast cancer incidence throughout Thailand (Virani et al., 2014).

In Thailand, the Ministry of Public Health has advised all health promotion hospitals (formerly known as health centers) to teach women aged from 25 and over how to do a breast self-examination. Women in reproductive age groups tend to be more aware of early detection measures, such as breast self-examination which could partly explain the significant increase in ASR through a wide range of birth cohort and also the mid-period of interest. Though breast self-examination cannot reduce breast cancer mortality (Lauby-Secretan et al., 2015), increasing and sustaining women awareness for clinical breast checkups and mammography can be expected in the setting of Thailand, as a middle income country.

After an exponential increase in young ages, agespecific-incidence rates of breast cancer plateaus at an age range around 10 years or more and is followed by a drop in older ages, however, it can be bimodal. In some Asian countries, there are two peaks where, the first peak is usually observed around the age of 40-60 and the second peak is at 85 plus years, while bimodal curve is not present in western countries it usually peaks at the age of 70 and over (CI5I-X, n.d.).

The age effect estimates of the AC-P model exhibit the well-known 'Clemmensen's hook' where incidence rates increase exponentially until around the age of 50 , then dip slightly before rising again at a lower rate. This phenomenon has been observed for breast cancer incidence and mortality across countries and is thought to be due to the overlap of rates between pre- and postmenopausal women (De Waard et al., 1964; Anderson, 1974; Bouchardy et al, 2006).

Compared to a high income country, such as the United States, where the ASR of breast cancer in CIV volume $\mathrm{X}$ was over 100 cases per 100,000 of the population (CI5I-X, n.d.). The ASR of breast cancer in Lampang is also relatively low in comparison to other low- and middle-income countries (LMICs). The ASRs from 1998 to 2002 in Korea, Taiwan, Hong Kong and Singapore were 37.2, 59.7, 69.1 and 90.1 cases per 100,000 of the population, respectively (Shin et al, 2010). Thailand is undergoing an epidemiologic transition in lifespan and lifestyle characteristics, thus, the increasing burden of breast cancer is observed in our analysis.

The risk profile and breast cancer screening of women in Northern Thailand was discussed elsewhere (Virani et al., 2014) and could be applied to women in the Northern region. In brief, the percentage of Southern Thai women who were overweight, having diabetes, or hypertension was found to be increasing by the two consecutive health examination surveys in 2004 and 2009. Considering parity, the total fertility rate in the Thai population has been gradually decreasing from 6 in 1970 to 3 in 1985, 2 in 1998 and 1.6 in 2010 (Prasartkul and Vapattanawong, 2012). This shift in risk profiles of Thai women makes it necessary to focus on the early detection and more awareness of breast cancer.

The change in the proportion of patients over the age of 50 from $67 \%$ in 2012 to almost $75 \%-84 \%$ in 2030 is an important phenomenon which, implies that postmenopausal patients are going to make up a higher proportion among breast cancer patients. This phenomenon is also true in Songkhla where the shift over time towards regional and localized stages was striking, and the proportion of distant metastatic cancers 
was gradually decreasing (Virani et al., 2014). It implies that the proportion of operable cases, and patients who need hormonal and chemotherapy treatment, is increasing as well. Therefore, the need for surgical and medical oncologists for breast cancer patients, and in fact, for other adenocarcinoma cancers will not only be a big constrain for health and medical care in Southern Thailand, but for the country as a whole.

In conclusion, breast cancer in the Nouthern region of Thailand has been, and will be increasing continuously with deceleration in rates throughout 2030 . The majority of the patients in the future are going to be among women aged 50 years and older. Primary prevention measures depend on the effectiveness of the control measures of metabolic disorder conditions, which is the general trend towards a western lifestyle.

Screening measures, and increased detection rates of early disease alone cannot reduce the increasing trend of the disease. And for these reasons the budget for treatment facilities, such as tertiary hospitals, with surgical facilities, and personnel in both surgical and medical oncology is going to be essential in the future.

\section{Acknowledgements}

We would like to thank Lampang cancer registry for information on breast cancer data. Dr. Sriplung acknowledges the support from the National Research University Fund, Prince of Songkla University (MED580635S).

\section{References}

Althuis MD (2005). Global trends in breast cancer incidence and mortality 1973-1997. Int J Epidemiol, 34, 405-412.

Anderson DE (1974). Genetic study of breast cancer: identification of a high risk group. Cancer, 34, 1090-7.

Bouchardy C, Morabia A, Verkooijen HM, et al (2006). Remarkable change in age-specific breast cancer incidence in the Swiss canton of Geneva and its possible relation with the use of hormone replacement therapy. BMC Cancer, $\mathbf{6}, 78$.

Carstensen B (2012). Statistical Analysis in the Lexis Diagram: Age-Period-Cohort Models. Gentofte, Denmark: Steno Diabetes Center.

Carstensen B, Plummer M, Laara E, Hills M (2013). Epi: a package for statistical analysis in epidemiology, R package version 1.1.49, CRAN.

CI5I-X (n.d.). from http://ci5.iarc.fr/CI5I-X/old/table4.asp?regi stry $=8402499$ andfemale $=2$ and volume $=1020032007$ andsub mit=Execute (accessed 5.8.15).

Colditz, G. and B. Rosner (2000). Cumulative risk of breast cancer to age 70 years according to risk factor status: data from the Nurses' Health Study. Am J Epidemiol, 152, 950964.

Cui X, Dai Q, Tseng M, Shu X-O, Gao Y-T, Zheng W (2007). Dietary Patterns and Breast Cancer Risk in the Shanghai Breast Cancer Study. Cancer Epidemiol Biomark Prev, 16, 1443-8.

Deerassamee S, Martin N, Sontipong S, et al (1999). Cancer in Thailand vol. II, 1992-1994, IARC Technical Report No.34, IARC Technical Report, Lyon, France: International Agency for Research on Cancer.

De Waard F, Baanders-Vanhalewijn EA, Huizinga J (1964).
The bimodal age distribution of patients with mammary carcinoma; Evidence for the existence of 2 types of human breast cancer. Cancer, 17, 141-51.

Doll R (1976). Comparison between registries. age- standardized rates, in: waterhouse jah, muir cs, correa p, powell $\mathrm{j}$ (eds.), cancer incidence in five continents, Vol. III (IARC Scientific Publications No. 15), Lyon, France: International Agency for Research on Cancer, 453-459.

Fan L, Zheng Y, Yu KD, et al (2009). Breast cancer in a transitional society over 18 years: trends and present status in Shanghai, China. Breast Cancer Res Treat, 117, 409-416.

Globocan 2012 - Home (n.d.). Available from http://globocan. iarc.fr/Default.aspx (accessed 4.8.15).

Henderson KD, Bernstein L, Henderson B, Kolonel L, Pike MC (2008). Predictors of the timing of natural menopause in the Multiethnic Cohort Study. Am J Epidemiol, 167, 1287-94.

National Cancer Institute (2013). Joinpoint regression program, Version 4.0.4, Statistical methodology and applications branch. Surveillance Research Program, National Cancer Institute.

Khuhaprema T, Attasara P, Sriplung H, Wiangnon S, Sangrajrang S (2013). Cancer in Thailand. Vol VII, 2007-2009, Bangkok, Thailand: National Cancer Institute.

Khuhaprema T, Attasara P, Sriplung H, Wiangnon S, Sumitsawan Y, Sangrajrang S (2012). Cancer in Thailand. Vol VI, 20042006, Bangkok, Thailand: National Cancer Institute.

Khuhaprema T, Srivatanakul P, Attasara P, et al (2010). Cancer in Thailand. Vol V, 2001-2003, Bangkok, Thailand: National Cancer Institute.

Khuhaprema T, Srivatanakul P, Sriplung H, et al (2007). Cancer in Thailand. Vol IV, 1998-2000, Bangkok, Thailand: Bangkok Medical Publisher.

Kim HJ, Fay MP, Feuer EJ, Midthune DN (2000). Permutation tests for joinpoint regression with applications to cancer rates. Stat Med, 19, 335-51.

Kruk J (2007). Association of lifestyle and other risk factors with breast cancer according to menopausal status: a case-control study in the Region of Western Pomerania (Poland). Asian Pac J Cancer Prev, 8, 513-524.

Lampang Province - Wikipedia (n.d.). from http://en.wikipedia. org/wiki/Lampang_Province (accessed 4.9.15).

Lauby-Secretan B, Scoccianti C, Loomis D, et al (2015). Breastcancer screening--viewpoint of the IARC Working Group. N Engl J Med, 372, 2353-8.

Layde PM, Webster LA, Baughman AL, et al (1989). The independent associations of parity, age at first full term pregnancy, and duration of breastfeeding with the risk of breast cancer. J Clin Epidemiol, 42, 963-973.

Mistry M, Parkin DM, Ahmad AS, Sasieni P. (2011) Cancer incidence in the United Kingdom: projections to the year 2030. Br J Cancer, 105, 1795-803.

National Statistical Office (2012). 2010 Population and housing census, Bangkok, Thailand: National Statistical Office.

National Statistical Office (2011). Population census, 2010, lampang, Bangkok, Thailand: national statistical office.

National Statistical Office (2002). 2000 Population and housing census, Bangkok, Thailand: National Statistical Office.

National Statistical Office (1992). Advance report: 1990 population and housing census, Bangkok, Thailand: national statistical office.

NORDPRED software package [Internet]. (2013) Oslo: Cancer Registry of Norway.

Office of the National Economic and Social Development Board (2013). Population projections for Thailand 2010-2040, Bangkok, Thailand: Office of the National Economic and Social Development Board.

Olsen AH, Parkin DM, Sasieni P (2008). Cancer mortality in the 
United Kingdom: projections to the year 2025,99, 1549-54.

Prasartkul P, Vapattanawong P (2012). Birth in: ThaiHealth 2012, Bangkok, Thailand: Institute for Population and Social Research, Mahidol University.

R Core Team (2014). R: A language and environment for statistical computing, Vienna, Austria: R Foundation for Statistical Computing.

Segi M (1960). Cancer mortality for selected sites in 24 countries (1950-57), Department of Public Health, Tohoku University of Medicine, Sendai, Japan.

Shin H-R, Joubert C, Boniol M, et al (2010). Recent trends and patterns in breast cancer incidence among Eastern and Southeastern Asian women. Cancer Causes Control, 21, 1777-85.

Sriplung H, Sontipong S, Martin N, et al (2003). Cancer in Thailand. Vol III, 1995-1997. Bangkok, Thailand: Bangkok Medical Publisher.

Sriplung H, Wiangnon S, Sontipong S, Sumitsawan Y, Martin N (2006). Cancer incidence trends in Thailand, 1989-2000. Asian Pac J Cancer Prev, 7, 239-244.

The World Bank (n.d.) Fertility rate, total (births per woman)

Thomas F, Renaud F, Benefice E, de Meeus T, Guegan JF (2001). International variability of ages at menarche and menopause: patterns and main determinants. Hum Biol, 73, 271-290.

Vatanasapt V, Martin N, Sriplung H, et al (1995). Cancer incidence in Thailand, 1988-1991. Cancer Epidemiol Biomark Prev, 4, 475-483.

Virani S, Sriplung H, Rozek LS, Meza R (2014). Escalating burden of breast cancer in southern Thailand: analysis of 1990-2010 incidence and prediction of future trends. Cancer Epidemiol, 38, 235-243. 